\title{
Relationship between Melatonergic and Thyroid Systems in Depression
}

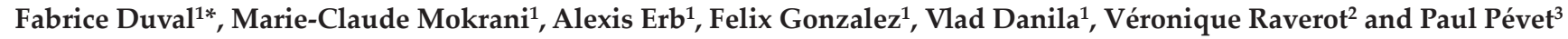 \\ ${ }^{1}$ Pôle 8/9 Psychiatry, APF2R, Centre Hospitalier, Rouffach, France \\ ${ }^{2}$ Service d'Hormonologie, Groupement Est, Hospices Civils de Lyon, France \\ ${ }^{3}$ UPR 3212 CNRS, Strasbourg University, France
}

${ }^{*}$ Corresponding author: Fabrice Duval, Centre Hospitalier, Pôle 8/9, APF2R, 27 rue du 4ème Spahis Marocain, 68250 Rouffach, France; Tel: 333-89-78-70-78; Fax: 333-8978-51-24; Email: f.duval@ch-rouffach.fr

Received: July 22, 2019; Accepted: July 30, 2019; Published: August 20, 2019;

\section{Summary}

Although melatonergic and thyroid system dysregulations are often observed in depression, it remains largely unknown whether these abnormalities are interrelated. Plasma melatonin concentrations were evaluated between $2100 \mathrm{~h}$ and $0800 \mathrm{~h}$ in $12 \mathrm{DSM}-5$ major depressed euthyroid inpatients; light (2,000 lux) was administered at midnight for one hour with a portable light device. On the following day, thyrotropin (TSH) responses to $0800 \mathrm{~h}$ and $2300 \mathrm{~h}$ protirelin (TRH) tests were measured. Melatonin profiles exhibited a wide interindividual variability. Light induced a reduction in melatonin concentrations. Melatonin suppression (MEL-S) values (expressed as percentage of change between concentration at midnight and lowest concentration after light) were correlated with $2300 \mathrm{~h}$ TRH-TSH responses ( $\Delta \mathrm{TSH}$ ) and $\triangle \Delta \mathrm{TSH}$ values (difference between $2300 \mathrm{~h}-\Delta \mathrm{TSH}$ and $0800 \mathrm{~h}-\Delta \mathrm{TSH})$. Post-light rise in melatonin (MEL-PLR) values (expressed as percentage of change between lowest concentration after light and concentration at $0400 \mathrm{~h}$ ) were correlated with $2300 \mathrm{~h}-\Delta \mathrm{TSH}$ and $\Delta \mathrm{TSSH}$ values. Moreover, patients with reduced $\Delta \Delta \mathrm{TSH}$ values $(<2.5 \mathrm{mU} / \mathrm{L})$ showed a trend toward lower MEL-S and MEL-PLR values than patients with normal thyroid function. Together, our results suggest close functional relationships between melatonergic and thyroid systems in depression.

Keywords: Depression, melatonin, light, Hypothalamic-Pituitary-Thyroid (HPT) axis, Thyrotropin-Releasing Hormone (TRH) test, thyrotropin (TSH).

\section{Introduction}

Depression is often associated with disrupted circadian rhythms. The neurohormone melatonin, synthesized by methylation of serotonin in the pineal gland, provides a robust circadian message to the organism (for review see Pevet, 2014) [1]. Given an inhibitory effect of light, melatonin secretion increases after dusk and terminates by dawn. It has been previously reported in healthy humans that melatonin secretion is suppressed at night with light of 500 lux or greater [2,3]. In depression it was found, but not unanimously, abnormalities of circadian rhythm of melatonin with advanced phases and/or decrease in nocturnal amplitude [4-6], and increased, decreased, or normal sensitivity to light [2].

On the other hand, while the vast majority of depressed patients have thyroid function tests within the euthyroid range, most inpatients exhibit a chronobiological Hypothalamic-Pituitary-Thyroid (HPT) axis dysregulation. Indeed, it has been consistently found that circadian thyrotropin (TSH) secretion is altered in depression (i.e., failure of the normal nocturnal surge of TSH, and lower and less variable 24-hour TSH levels compared to controls [5]) associated with blunted TSH response to protirelin $(\mathrm{TRH})$ and reduced difference in TSH response between $2300 \mathrm{~h}$ and $0800 \mathrm{~h}$ TRH tests $(\Delta \Delta \mathrm{TSH}$ ) (for review see Duval and Mokrani, 2018) [7]. In depression, the $\Delta \Delta \mathrm{TSH}$ test represents a very sensitive chronobiological index since it is reduced in about $70 \%$ of inpatients [7].
Preclinical studies have demonstrated complex interactions between melatonergic and HPT systems. Different experimental models suggest inhibitory effects of melatonin on thyroid secretion $[8,9]$. In humans, it has been hypothesized that melatonin could act on the HPT axis by a "feed-sideward" effect that diminishes or increases responses when stimuli are respectively too high or too low [10]. To date, very few studies have focused on the links between melatonergic and HPT systems in depressed patients. Souêtre et al. [5] reported a positive correlation between amplitudes of melatonin and TSH circadian rhythms. Kjellmann et al. [11] found no significant correlation between nocturnal melatonin and morning $\Delta$ TSH levels.

The main goal of our exploratory pilot study was to investigate the possible pineal-thyroid functional relationship in euthyroid depressed inpatients, by examining in the same subjects the melatonin response to light administered at midnight, and the TSH response to TRH challenge at 0800 and $2300 \mathrm{~h}$, performed on the following day.

\section{Methods and Material}

\section{Subjects}

Twelve inpatients ( 5 male and 7 female; aged 38 to 58 years; mean age \pm SD, $50.6 \pm 6.2$ years) meeting DSM-5 criteria for major depressive disorder participated in this study. They were recruited from the inpatient units of the Pole 8/9, Psychiatric Hospital of 
Rouffach (France). None had a history of recent suicidal behavior (in 5 patients the last suicide attempt occurred $>1$ year prior to evaluation). They were free of all drugs for a minimum of 1 week; this washout was supervised in hospital. Patients were evaluated by means of at least two unstructured clinical interviews conducted by an experienced research psychiatrist (F.G.L., or V.D.) and a structured interview (Schedule for Affective Disorders and SchizophreniaLifetime Version)—conducted by a separate psychiatrist (A.E). The final diagnoses were made by consensus of two psychiatrists blind to endocrine results. Severity of depression was measured with the 17item Hamilton Rating Scale for Depression (HAM-D); inclusion in the study required a baseline HAM-D of 18 or greater (mean \pm SD, $23.5 \pm 4.0)$.

The protocol was approved by the local ethical committee (Comité de Protection des Personnes Est IV, Hôpital Civil Strasbourg) and performed according to the Declaration of Helsinki. All participants gave their informed and written consent. Routine blood tests and physical examination excluded subjects with medical illnesses. All patients had normal basal thyroid hormone values (TSH, free $\mathrm{T}_{4}\left[\mathrm{FT}_{4}\right]$ and free $\left.\mathrm{T}_{3}\left[\mathrm{FT}_{3}\right]\right)$, and normal body mass index $(18.5<\mathrm{BMI}<24.9$ $\mathrm{kg} / \mathrm{m}^{2}$ ). Subjects with a history of thyroid or other endocrine diseases; alcoholism or drug abuse; previous treatment with lithium salts, carbamazepine, long-acting neuroleptics, fluvoxamine, monoamine oxidase inhibitors or electroconvulsive therapy within one year of testing; and women taking oral contraceptives were excluded. We also excluded from the study patients with ocular disease, including cataract, glaucoma, retinitis pigmentosa, diabetic retinopathy, macular degeneration, or Stargardt disease.

All subjects were on a caffeine-restricted diet for at least three days before testing and their environment was synchronized, with diurnal activity from $0800 \mathrm{~h}$ to $2300 \mathrm{~h}$, and nocturnal rest (sleep).

\section{Procedures and Measurements}

Light was administered with Luminettes (Lucimed Company, Villers-le-Bouillet, Belgium). This portable LED device precisely focuses the light rays onto the lower half of the retina no matter the incline angle of the eye. During the session, performed in a room lighted below 50 lux, subjects were sitting in their bed. Light (2000 lux) was administered at midnight for one hour using Luminettes ${ }^{\circ}$. Subjects could sleep after light administration, but only in a sitting position to eliminate the influence of posture on melatonin levels. The intensity of 2000 lux and the duration of light exposure (one hour) were chosen to obtain a significant degree of melatonin suppression [3]. An indwelling cannula was inserted at $2000 \mathrm{~h}$ into a forearm vein and kept open with a slow drip of heparinized saline (5,000 IU heparin/l). Blood was sampled for melatonin determination at $2100 \mathrm{~h}$, and then every 20 minutes between $2320 \mathrm{~h}$ and $0500 \mathrm{~h}$, a last sample was taken at $0800 \mathrm{~h}$.

On the following day, the first TRH stimulation test was carried out at $0800 \mathrm{~h}$-blood was drawn for assay of TSH at -15, 0, 15, 30, 60 minutes after injection of $200 \mu \mathrm{g}$ TRH intravenously (TRH Ferring ${ }^{\circ}$, Ferring Pharmaceuticals, Kiel, Germany) - and the second TRH test was performed at $2300 \mathrm{~h}$, on the same day, using the same previously depicted procedure [12]. Given that high cortisol secretion could decrease melatonin production, a Dexamethasone Suppression Test (DST) was carried out at midnight with oral ingestion of $1 \mathrm{mg}$ of dexamethasone (Dectancyl, Laboratoires Roussel, Paris, France) followed by the assay of plasma cortisol at $0800 \mathrm{~h}, 1600 \mathrm{~h}$ and 2300 $\mathrm{h}$ on the next day. After the tests, blood samples were immediately centrifuged at $3000 \mathrm{rpm}$ and $4^{\circ} \mathrm{C}$; plasma samples were then stored at $-20^{\circ} \mathrm{C}$ until assay.

Hormonal concentrations were determined by Radioimmunoassay (RIA) techniques (melatonin) and immunometric techniques based on enhanced luminescence (TSH, cortisol). Average intra-assay and inter-assay coefficients of variation were respectively: melatonin: $<7 \%-8 / 3 \%$, sensitivity < 3 ng/L [3,4]; TSH: $3.4 \%-4.8 \%$, sensitivity $<0.01 \mathrm{mU} / \mathrm{L}$ (Access Hypersensitive hTSH Assay, Beckman Coulter, Inc., Fullerton, CA, USA); Cortisol: $5.1-6.8 \%$, sensitivity $<11 \mathrm{nmol} / \mathrm{L}$ (Access Cortisol Assay, same supplier).

\section{Results}

Melatonin profiles exhibited a wide interindividual variability. Light induced a reduction in melatonin concentrations and lowest values were observed at $0113 \mathrm{~h} \pm 30$ minutes (mean $\pm \mathrm{SD}$ ). Melatonin suppression (MEL-S) to light was expressed as percentage of change between concentration at midnight and lowest concentration after light according to the formula: MEL-S $=$ ([melatonin concentration at $2400 \mathrm{~h}$ (immediately prior to phototherapy; i.e., baseline) - minimum melatonin concentration after light]/melatonin concentration at 2400 h) $\times-100$; mean \pm SD, $31.9 \pm 23.5 \%$. Post-light rise in melatonin (MEL-PLR) was expressed as percentage of change between lowest concentration after light and melatonin value at $0400 \mathrm{~h}$ according to the formula: MEL-PRL $=([$ minimum melatonin concentration after light (i.e., baseline) - melatonin concentration at $0400 \mathrm{~h}] /$ minimum melatonin concentration after light $) \times 100$; mean \pm SD, $102 \pm 103 \%$. The calculation of the percentage of melatonin changes (during and after light exposure) eliminated the baseline effect, although the intersubject variations remained very large.

MEL-S and MEL-PLR values were not significantly correlated $\left(r_{S}\right.$ $[$ Spearman] $=0.43, \mathrm{n}=12, \mathrm{p}=0.16)$. However, there was a positive relationship between MEL-S and MEL-PLR values and evening TSH responses to TRH (Figure 1)-expressed as the maximum increment above the baseline value after TRH injection (2300h- $\Delta$ TSH), and the difference between $2300 \mathrm{~h}-\Delta \mathrm{TSH}$ values and $0800 \mathrm{~h}-\Delta \mathrm{TSH}(\Delta \Delta \mathrm{TSH})$. Correlations between MEL-S and MEL-PLR and 0800h- $\Delta$ TSH values were not significant. In our population, MEL-S, MEL-PLR, and TSH (baseline, $\Delta \mathrm{TSH}$, and $\Delta \Delta \mathrm{TSH}$ ) values were unrelated to age, gender, severity of depression and post-DST cortisol values.

We further classified the patients on the basis of their $\Delta \Delta$ TSH test status, since reduced $\Delta \Delta \mathrm{TSH}$ values reflect chronobiological HPT axis alterations (for review see Duval and Mokrani, 2018) [7]. The first group showed reduced $\Delta \Delta \mathrm{TSH}$ values (i.e. less than $2.5 \mathrm{mU} / \mathrm{L}$ [Duval et al. 2015]) ( $\mathrm{n}=5 ; 3$ male and 2 female; mean age, $51.6 \pm$ 3.1 years). The second group showed normal $\Delta \Delta$ TSH values $(n=7$; 2 male and 5 female; mean age, $49.9 \pm 7.9$ years). Statistical analysis employing generalized Friedman rank sum test for rough melatonin 
data (sampled between $2320 \mathrm{~h}$ and $0500 \mathrm{~h}$ ) showed a significant time effect in patients with normal $\Delta \Delta$ TSH values $\left(\mathrm{p}<0.910^{-7}\right)$, but not in patients with reduced $\Delta \Delta \mathrm{TSH}$ values $(\mathrm{p}=0.11)$. MEL-S and MEL-
PLR values tended to be lower in patients with reduced $\Delta \Delta \mathrm{TSH}$ values compared to patients with normal HPT activity (Figure 2).

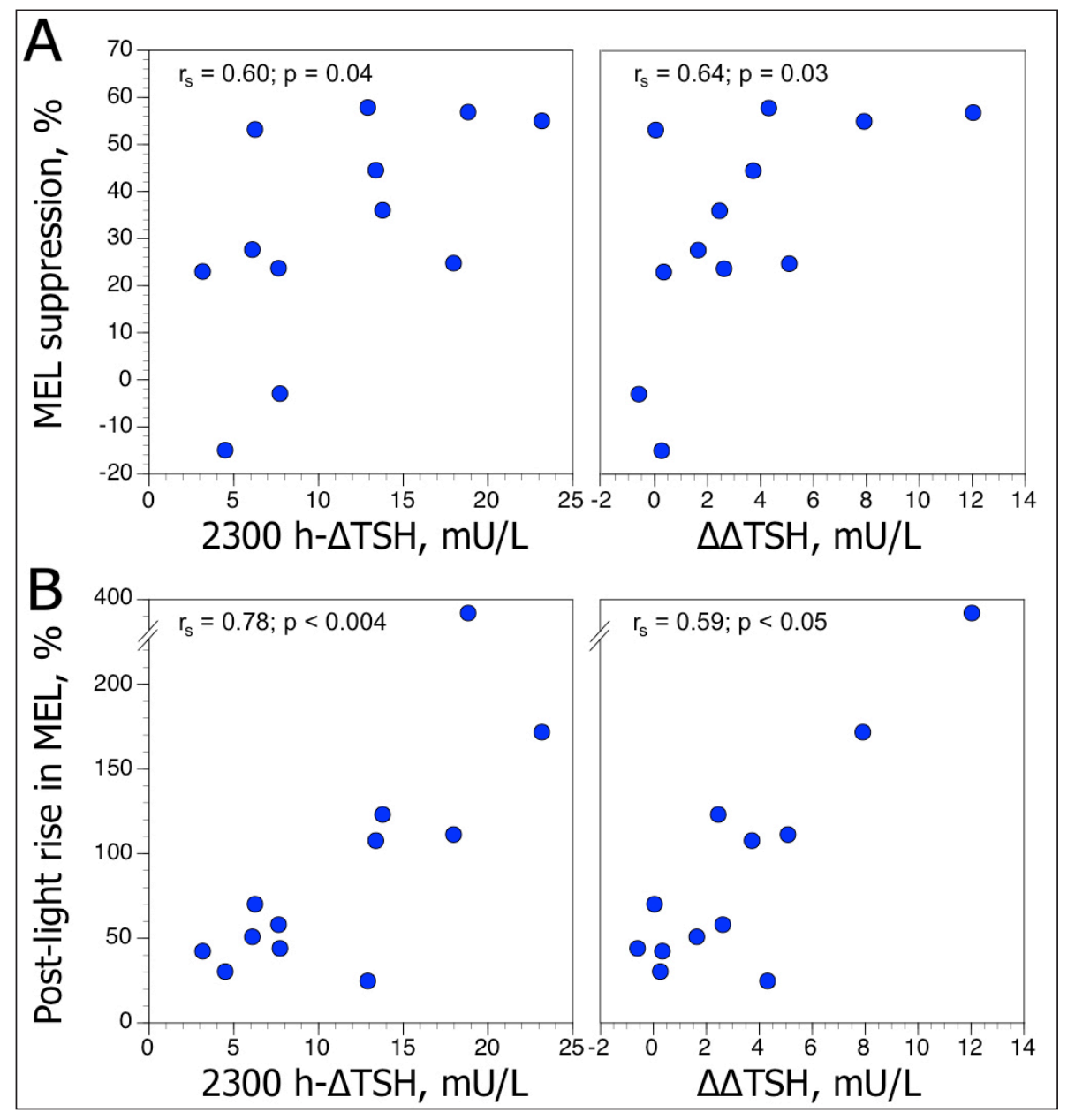

Figure 1. Relationships between maximum increment in plasma TSH level above baseline ( $\Delta \mathrm{TSH})$ after TRH injection at $2300 \mathrm{~h}$, and difference between $2300 \mathrm{~h}-\Delta \mathrm{TSH}$ and $0800 \mathrm{~h}-\Delta \mathrm{TSH}$ values $(\Delta \Delta \mathrm{TSH})$, and (A) melatonin suppression (MEL-S) to light, expressed as percentage of change between concentration at midnight and lowest concentration after light, and (B) post-light rise in melatonin (MEL-PLR), expressed as percentage of change between lowest concentration after light and melatonin value at $0400 \mathrm{~h}$, in 12 depressed patients. $\mathrm{r}_{\mathrm{s}}$ : Spearman rank coefficient.

\section{Discussion}

The main findings of this study are as follows: (1) light-induced melatonin amplitude suppression and post-light rise in melatonin are positively correlated to nocturnal TRH-TSH responses (i.e, $2300 \mathrm{~h}-\Delta \mathrm{TSH}$ and $\Delta \Delta \mathrm{TSH}$ ) in depressed patients; and (2) patients with HPT axis dyregulation show a trend toward decreased melatonin responses to light. The present results provide some evidence that melatonergic and thyroid systems are interrelated, suggesting the involvement of common mechanisms.

Pineal and thyroid activity follows a circadian rhythm closely connected to the hypothalamic Suprachiasmatic Nuclei (SCN), which house the master circadian clock. In preclinical studies SCN ablation resulted in marked alteration in both melatonin and HPT axis hormones rhythms. Thus, a downward trend in nocturnal responses of melatonin (to light) and TSH (to TRH) could possibly result from a weakened output of the SCN in depression. Moreover, since reduced $\Delta \Delta \mathrm{TSH}$ values may reflect altered TRH receptor chronesthesy on pituitary thyrotrophs secondary to endogenous TRH hypersecretion (for review see Duval and Mokrani, 2018) [7], our findings could also suggest that decreased melatonin function may act at the hypothalamus by disinhibiting TRH release. Indeed, some animal studies reported that decreased pineal activity enhanced secretion of TRH, while injected melatonin altered the secretion and production of TRH $[13,14]$. Conversely, in vitro studies demonstrated that TRH could antagonize the effects of melatonin [15]. Therefore, another intriguing possibility is that increased TRH secretion in depression could lead to altered melatonin functionality. These latter two hypotheses are not mutually exclusive. However, future studies are needed to confirm these assumptions. 


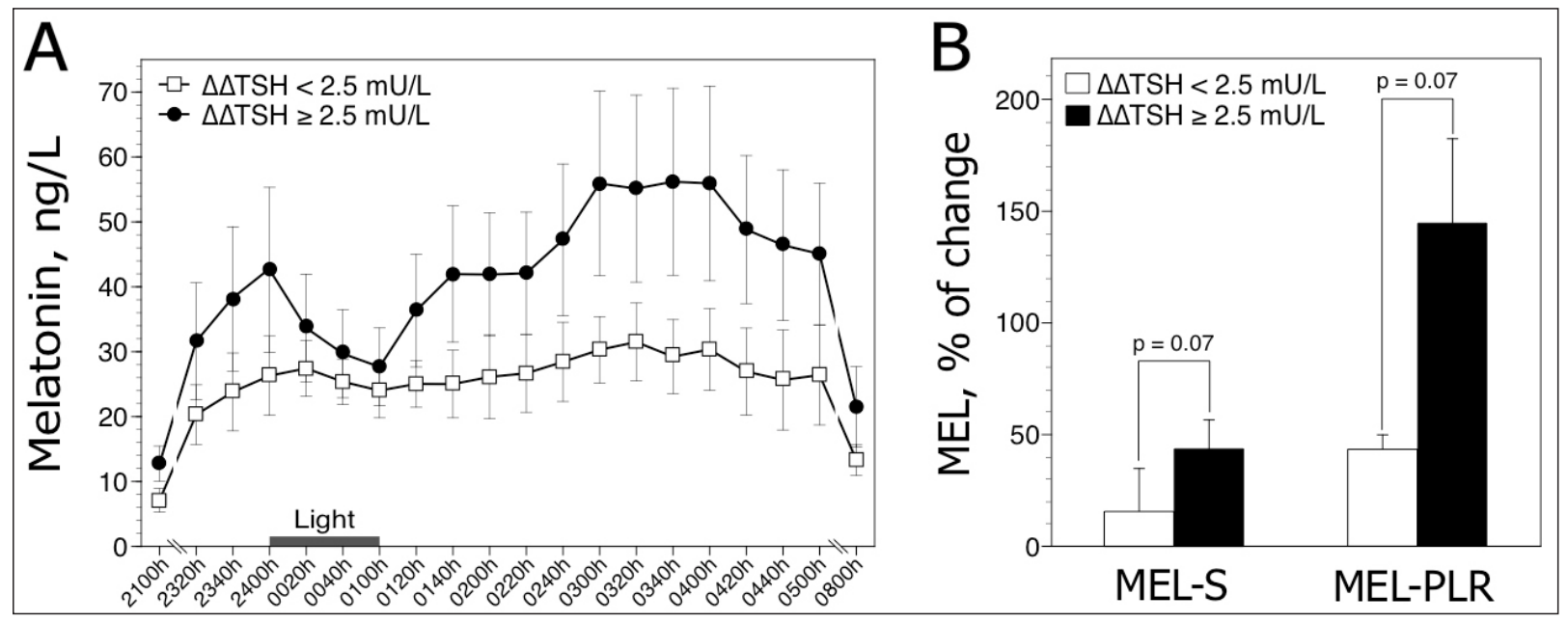

Figure 2. Nocturnal melatonin profiles (A) and responses to light (B) in 12 depressed patients classified according to the presence $(\Delta T S H<2.5 \mathrm{mU} / \mathrm{L}$, $\mathrm{n}=5)$ or absence $(\Delta \Delta \mathrm{TSH} \geq 2.5 \mathrm{mU} / \mathrm{L}, \mathrm{n}=7)$ of HPT dysregulation. Raw data are expressed as mean $\pm \mathrm{SEM}$. The histograms $( \pm \mathrm{SEM})$ represent the mean melatonin suppression (MEL-S) and post-light rise in melatonin (MEL-PLR). P values are obtained by Mann-Whitney two-tailed test.

\section{Limitations of the Present Study}

Some shortcomings in our study require discussion. Firstly, owing to the lack of healthy comparison subjects we cannot demonstrate that plasma melatonin levels before and after light exposure are abnormal in depression. However, it is noteworthy that we used the same melatonin radioimmunoassay as Claustrat et al. [3] who administered light with eyeglass LED delivery systems (Somnavue ${ }^{\circ}$ and Lumino ${ }^{\circ}$ ) in 10 healthy individuals; in comparison, the mean melatonin profile of our patients appears diminished. Secondly, given the exploratory nature of our research we studied a rather small sample of depressed inpatients. This may have reduced the statistical power of our analyses (performed with nonparametric methods) since results, although suggestive of an impaired functionality of the melatonergic system in patients with abnormal HPT axis activity, did not achieve statistical significance. Thus, our findings must be considered preliminary until replicated in a larger patient population.

In conclusion, our pilot study suggests that pineal and thyroid systems exert mutual interregulation. In the future, it will be important to understand the mechanisms underlying links between melatonin, HPT axis, circadian rhythms, and sleep-wake regulation in order to provide novel insight into the pathophysiology of affective disorders.

\section{Aknowledgment}

The authors express their gratitude to the nurses of the pole $8 / 9$, Centre Hospitalier, Rouffach, France

\section{Role of the Funding Source}

Funding of this study was provided by inner sources (Association Pour la Formation et la Recherche de Rouffach [APF2R], Centre Hospitalier, Rouffach). No outside parties had any role in study design; in the collection, analysis, and interpretation of data; in the writing of the report and in the decision to submit the paper for publication.

\section{References}

1. Pévet P (2014) the internal time-giver role of melatonin. A key for our health. Rev Neurol (Paris) 170: 646-652.
2. Nurnberger JI, Adkins S, Lahiri DK, Mayeda A, Hu K, Lewy A, et al (2000) Melatonin suppression by light in euthymic bipolar and unipolar patients. Arch Gen Psychiatry 57: 572-579.

3. Claustrat B, Brun J, Borson-Chazot F, Cohen-Tannoudji D, Claustrat F, et al (2010) Suppression of melatonin secretion in healthy subjects with eyeglass LED delivery system. Neuro Endocrinol Lett 31, 330-335.

4. Claustrat B, Chazot G, Brun J, Jordan D, Sassolas G (1984) A chronobiological study of melatonin and cortisol secretion in depressed subjects: plasma melatonin, a biochemical marker in major depression. Biol Psychiatry 19: 1215-1228.

5. Souêtre E, Salvati E, Belugou JL, Pringuey D, Candito M, et al (1989) Circadian rhythm in depression and recovery: evidence for blunted amplitude as the main chronbiological abnormality. Psychiatry Res 20: 263-278.

6. Crasson M, Kjiri S, Colin A, Kjiri K, L'Hermite-Baleriaux M, et al (2004) Serum melatonin and urinary 6-sulfatoxymelatonin in major depression. Psychoneuroendocrinology 29: 1-12.

7. Duval F, Mokrani MC (2018) Thyroid axis activity in depression. Ann Thyroid Res 4: 166-171.

8. Wright ML, Cuthbert KL, Donohue MJ, Solano SD, Proctor KL (2000) Direct influence of melatonin on the thyroid and comparison with prolactin. $J$ Exp Zool 286: 625-631.

9. Prendergast BJ, Pyter LM, Kampf-Lassin A, Patel PN, Stevenson TJ (2013) Rapid induction of hypothalamic iodothyronine deiodinase expression by photoperiod and melatonin in juvenile Siberian hamsters (Phodopus sungorus). Endocrinology 154: 831-841.

10. Mazzoccoli G, Giuliani A, Carughi S, De Cata A, Puzzolante F (2004)The hypothalamic-pituitary-thyroid axis and melatonin in humans: possible interactions in the control of body temperature. Neuro Endocrinol Lett 25: 368-372.

11. Kjellman BF, Ljunggren JG, Beck-Friis J, Wetterberg L (1985) Effect of TRH on TSH and prolactin levels in affective disorders. Psychiatry Research 14: 353-363.

12. Duval F, Mokrani MC, Erb A, Gonzalez Lopera F, Alexa C, et al (2015) Chronobiological hypothalamic-pituitary-thyroid axis status and antidepressant outcome in major depression. Psychoneuroendocrinology 59: 71-80.

13. Relkin R (1978) Use of melatonin and synthetic TRH to determine site of pineal inhibition of TSH secretion. Neuroendocrinology 25: 310-318.

14. Mitsuma T, Nogimori T (1985) Effects of various drugs on thyrotropin secretion in rats. Horm Metab Res 17: 337-341.

15. Naftalin RJ, Cunningham P, Afzal-Ahmed I (2004) Piracetam and TRH analogues antagonise inhibition by barbiturates, diazepam, melatonin and galanin of human erythrocyte D-glucose transport. Br J Pharmacol 142: 594-608.

\section{Citation:}

Fabrice Duval, Marie-Claude Mokrani, Alexis Erb, Felix Gonzalez, Vlad Danila, Véronique Raverot and Paul Pévet (2019) Relationship between Melatonergic and Thyroid Systems in Depression. Endocrinol Diabetes Metab J Volume 3(5): $1-4$. 